\title{
Open Partial Nephrectomy: One Night Length of Stay Is Safe and Cost Effective
}

\author{
Mohit Sirohi, Kyrollis Attalla, Harris M. Nagler, Erik T. Goluboff* \\ Mount Sinai Beth Israel, New York, USA \\ Email: *egoluboff@chponet.org
}

How to cite this paper: Sirohi, M., Attalla, K., Nagler, H.M. and Goluboff, E.T. (2016) Open Partial Nephrectomy: One Night Length of Stay Is Safe and Cost Effective. Open Journal of Urology, 6, 139-146. http://dx.doi.org/10.4236/oju.2016.69023

Received: June 30, 2016

Accepted: September 11, 2016

Published: September 14, 2016

Copyright $\odot 2016$ by authors and Scientific Research Publishing Inc. This work is licensed under the Creative Commons Attribution International License (CC BY 4.0).

http://creativecommons.org/licenses/by/4.0/

\section{(c) (i) Open Access}

\begin{abstract}
Objective: To review our open partial nephrectomy (OPN) experience and compare to known robotic partial nephrectomy (RPN) data to determine whether length of stay (LOS) and morbidity are significant drivers in the surgical approach employed for partial nephrectomy. Methods: We reviewed our OPN experience during the last 3 years examining age, tumor size, LOS, pathology, blood loss, complications, recurences, and deaths. Results: Seventy-five patients underwent OPN during this period. Mean age was 59 years, tumor size $2.8 \mathrm{~cm}$, percent malignant $75 \%$, estimated blood loss $350 \mathrm{cc}$. With a median follow-up of 18 months, there was one urinoma managed by drainage, one pseudo aneurysm that required embolization and one pulmonary embolism that required anticoagulation. There were no readmissions, no tumor recurences, and no deaths. Our major complication rate was $4 \%$ as compared to other trials that reported major complication rates between $1 \%-9 \%$ for RPN and between $3 \%-24 \%$ for OPN. In the first half of the experience $(n=37)$, median LOS was 57 hours. Using a pathway encouraging early ambulation and smaller incisions in the second half of the experience $(\mathrm{n}=38)$, median LOS was 35 hours. This is much shorter than reported RPN LOS of $62-67$ hours and OPN LOS of $108-142$ hours. Conclusion: OPN can be performed safely and effectively with one night hospital stay. This provides a more cost-effective approach to partial nephrectomy with similar or better complication rates and calls into question the main value drivers of RPN.
\end{abstract}

\section{Keywords}

Cost Effective, Length of Stay, Open Partial Nephrectomy, Robotic Partial Nephrectomy

\section{Introduction}

Partial nephrectomy rates continue to increase as the importance of nephron preserva- 
tion is recognized for maintaining renal function without compromising oncologic outcomes [1]-[3]. Nephron sparing surgery is the recommended treatment [4] for small renal masses; this operation was initially performed through an open approach. However, with the advent of laparoscopy, urologists began operating on renal masses through a minimally invasive approach. In recent years, robotic technology has been advocated by some to be advantageous.

The use of the robot has given surgeons a new formidable tool to perform once technically challenging laparoscopic procedures, such as partial nephrectomies [5]. However, the learning curve for use on the robot is still relatively steep and senior or novice surgeons face the challenge of either learning the practice, referring a patient to a robotic surgeon, or performing a traditional open partial nephrectomy (OPN).

As we move into an era of medicine where health care costs are shifted from fee for service to risk models, cost drivers will be increasingly scrutinized. Robotic procedures are associated with increased capital and operational costs. Proponents of robotic partial nephrectomy (RPN) claim shorter hospital length of stay (LOS) and less morbidity as compared to OPN as justification for the increased costs of using this technology. Quite possibly, these claims, along with other post-operative measures, sway providers away from performing OPN. We reviewed our OPN experience in a community teaching hospital to determine whether LOS and decreased morbidity could still be significant drivers in the surgical approach to partial nephrectomy employed by urologists.

\section{Methods}

Institutional Review Board approval was obtained before the commencement of this study. A retrospective review of the data was collected from the hospital paper and electronic database. OPN patient information was collected between August 2011 and June 2014 using the hospital's procedure coding system as well as through a resident universal log. All open partial nephrectomies performed by a single surgeon (ETG) were included in the study. Exclusion criteria included any case that began as laparoscopic or RPN. Patient demographics, operative data, estimated blood loss (EBL) and laterality, tumor pathology, and post-operative data including LOS in hours, thirty-day post-operative major complications utilizing the Clavien-Dindo classification system [6], post-operative pathway, recurrences, and death were collected.

Patients were grouped into the first or second half of the cohort after a shift in the pre, intra-and post-operative management pathway of the patients halfway through our study. The major points of this new rapid discharge pathway included: 1) a modified "mini flank" incision [7], as small as feasible to reduce post-operative pain while still maintaining hilar and oncologic control; 2) emphasis on the importance of early ambulation and safe early discharge; 3 ) preservation of the extra peritoneal approach; and 4) Jackson-Pratt (JP) drainage postoperatively, regardless of entry into the collecting system.

Post-operatively, patients are counseled that night to sit out of bed to a chair and ambulate the night of surgery or on POD one. The Foley catheter is removed overnight 
allowing for easier ambulation and earlier trial of void. All labs are drawn immediately post-operatively and at midnight to facilitate formulating a thorough decision on morning rounds. The JP drain was removed depending on output $(<10 \mathrm{cc} / \mathrm{hr})$ just prior to discharge home. If the output was greater than $10 \mathrm{cc} / \mathrm{hr}$, fluid from the drain was sent for analysis for creatinine. All stable patients had their Foley catheters removed at midnight on post-operative day (POD) zero. Patients were switched from intravenous (IV) patient controlled analgesia (PCA) to oral pain medication on POD one. All patients had a basic metabolic panel and complete blood count drawn immediately postoperatively and at midnight of POD zero. Once patients are tolerating a regular diet, ambulating, and pain is controlled, they are discharged with a one week follow up in the office and called at home the day after discharge. Patients are discharged home with oral pain medication, and stool softener. We believe that seeing or speaking with the patient the evening after surgery for counseling to sit out of bed and ambulate is a simple, yet effective measure to ensure an early discharge and avoid complications. Properly counseled team members are all valuable in the patient's outcome.

Other aspects of this pathway included changes in diet orders. Depending on entry into the peritoneal space, patients were placed on a clear liquid diet (entry) or regular diet (no entry) on POD zero. They were encouraged to ambulate on POD zero and one. The JP drain was removed depending on output $(<10 \mathrm{cc} / \mathrm{hr})$ just prior to discharge home. If the output was greater than $10 \mathrm{cc} / \mathrm{hr}$, fluid from the drain was sent for analysis for creatinine. All stable patients had their Foley catheters removed at midnight on post-operative day (POD) zero. Patients were switched from intravenous (IV) patient controlled analgesia (PCA) to oral pain medication on POD one. All patients had a basic metabolic panel and complete blood count drawn immediately post-operatively and at midnight of POD zero. Patients were discharged once they tolerated a regular diet, ambulating, and pain was controlled.

A paired t-test was used to determine differences in mean age, EBL, tumor size and mean and median lengths of stay between the two cohorts.

\section{Results}

A total of seventy five patients underwent an OPN from August 2011 and June 2014. Mean age was 59 years, mean tumor size $2.8 \mathrm{~cm}$, mean estimated blood loss was $351 \mathrm{cc}$, and mean length of stay was 48.7 hours. Seventy five percent of the tumors were malignant. Median follow up was 18 months. There was one urinoma managed by percutaneous drainage, one pseudo aneurysm that required embolization, one pulmonary embolism that required anticoagulation, no tumor recurrences, and no deaths. The total major complication rate (Clavien grade III-V) was $4 \%$. There were 37 patients in the first half, from August 2011 to October 2012, and 38 in the second half from October 2012 to June 2014 Table 1.

When comparing the first half patients with the second (rapid discharge pathway), there was no significant difference in age $(p=0.204)$, EBL $(p=0.938)$, or tumor size $(\mathrm{p}=0.575)$. The major complications included one from the first half and two from the 
Table 1. Patient demographics.

\begin{tabular}{cccc}
\hline Patient Demographics & Group 1 $(\mathrm{n}=37)$ & Group 2 (n=38) & $\begin{array}{c}\text { t-test (2 sample unequal } \\
\text { variance, 2-tail) }\end{array}$ \\
\hline Mean Age (yrs) & 56.6 & 60.5 & $\mathrm{p}=0.204$ \\
Average LOS (hrs) & 62.2 & 48.7 & $\mathrm{p}=0.049$ \\
Median LOS (hrs) & 56.6 & 35.3 & \\
Mean EBL (mL) & 355 & 348 & $\mathrm{p}=0.938$ \\
Mean Tumor Size (cm) & 2.92 & 2.72 & $\mathrm{p}=0.575$ \\
Laterality & R: $16, \mathrm{~L}: 21$ & R: $23, \mathrm{~L}: 15$ & \\
Gender & M: 25, F: 12 & M: 29, F: 9 &
\end{tabular}

second. There was a statistically significant difference in mean and median lengths of stay between the two groups (62.2 versus 48.7 hours and 56.6 and 35.3 hours, respectively, first group versus second, $\mathrm{p}<0.05)$.

\section{Discussion}

As rates of partial nephrectomy continue to rise, patients with renal masses amenable to a partial excision will continue to seek guidance from their urologists as to the best surgical approach. Traditionally, these patients would have undergone an OPN as the definitive surgical treatment option. However, with increased use of the robot, more patients are operated on through a minimally invasive robotic approach with similar perioperative, oncological and functional outcomes as seen with OPN [8]. Between 1998 and 2010, almost $85 \%$ of partial nephrectomies were performed through an open approach and almost $8 \%$ through a robotic approach [9]. However, if re-examined between 2008 and 2010, the percentage of OPN dropped to $67 \%$ and the percentage of RPN rose to nearly $24 \%$. This reflected a relative annual increase in OPN of $8 \%$ versus $45 \%$ for RPN.

Not surprisingly, a higher percentage of RPN were performed at an urban location (98.1\%) compared to OPN (94.3\%). This paper also highlighted that more patients had a prolonged length of stay (greater than 75 hours) in the OPN group than the RPN group (34.8\% vs. $12.4 \%$; Adjusted OR of 0.27 of RPN vs. OPN). Some have concluded that increasing partial nephrectomy rates are enabled due to robotic technology availability [10] but this still remains a controversial topic. Conceivably the reason for the sharp increase in RPN is due to a shift of minimally invasive surgeons from the laparoscopic approach to the robotic one, or simply patient demand.

One cannot argue that many patients who would have once been operated on through an open approach are now being referred to outside institutions for robotic treatment, and this number will only continue to rise. Although it is largely driven by patient preference, urologists may be swayed by data that states that RPN holds an advantage as it relates to length of stay, and hospitals may justify the use of robotic technology based on cost due to the reported differences in lengths of stay. Here, we have examined a driver for the recent and continued future shift length of stay, in an attempt 
to show that even at a relatively low volume center, OPN can still safely be performed with a one night length of stay.

Our data indicates that in our rapid discharge pathway arm $(n=38)$, with a median length of stay of 35.3 hours, two readmissions, and only two major complications, and no differences seen in the other variables of age, tumor size and EBL, OPN can be performed safely with a one night length of stay. Utilizing data from more recent publications, such as Mano et al. [11], which evaluated the cost of RPN versus OPN based primarily on length of stay and operating room expense, this amounts to a much more cost effective approach to partial nephrectomies than RPN. They found that RPN was, on average, $\$ 3765$ more costly when examining pure surgical costs and $\$ 1126$ cheaper when examining pure hospital cost, largely based on length of stay. The study reported a median difference of 1 day LOS ( 2 days for OPN versus 1 day for RPN) between the OPN and RPN groups and concluded that OPN, despite the longer LOS, was \$2539 cheaper, on average than RPN. While the authors specifically compared the cost of open and RPN utilizing a short postoperative pathway at a major oncologic center, no study has examined the role of a specific pathway in a moderately low volume center when evaluating LOS. Although the tumors were slightly larger $(3 \mathrm{~cm}$ vs. $2.7 \mathrm{~cm})$ and possibly more complex than those operated on at our institution, our LOS was 35 hours, compared to their LOS of a median of 2 days for the open approach vs. 1 day for the robotic approach. This further justifies their conclusion that the cost of an OPN is lower than a RPN when exercising a streamlined pathway.

It has also been presented that when comparing the costs of RPN, laparoscopic partial nephrectomy (LPN), and OPN, the cost of the robotic approach can be equivalent to the open approach only by minimizing both operating room time and LOS [12]. However, no study to the best of our knowledge, has displayed lower operating room time for RPN versus OPN. Mean reported hospitalization times of RPN have ranged from one to five days vs. OPN times ranging from two to seven days [1] [2] [8] [11][14]. When comparing OPN vs. LPN vs. RPN, Mir et al. concluded that LPN was most cost-effective due to the greatest balance between LOS and equipment cost [15]. That meta-analysis found that the LOS of OPN, LPN and RPN was 5.9, 3.2, 2.6 days, respectively. However, it has been argued that many of the studies were at large centers and the training time for laparoscopic surgery is reportedly much longer than robotic surgery, thus negating many of the LOS advantages [16]. While robotic assisted radical prostatectomy (RARP) has been shown to have higher hospitalization costs compared to open radical prostatectomy (ORP), there are higher in-hospital reimbursements for RARP compared to ORP (about $\$ 2000$ more per case) for privately insured younger patients [17]. As far as we know, it is unclear how hospital reimbursement for RPN compares to OPN, especially when comparing length of stay and complication rate.

Perhaps the robotic approach's benefits of pain and hence, LOS, do not apply if patients are thoroughly counseled. Our rapid discharge pathway is composed of several key pre, intra, and post-operative steps to ensure a safe outcome and early discharge (Figure 1). Patients are counseled pre-operatively that they will likely remain in the 


\section{RAPID DISCHARGE PATHWAY}

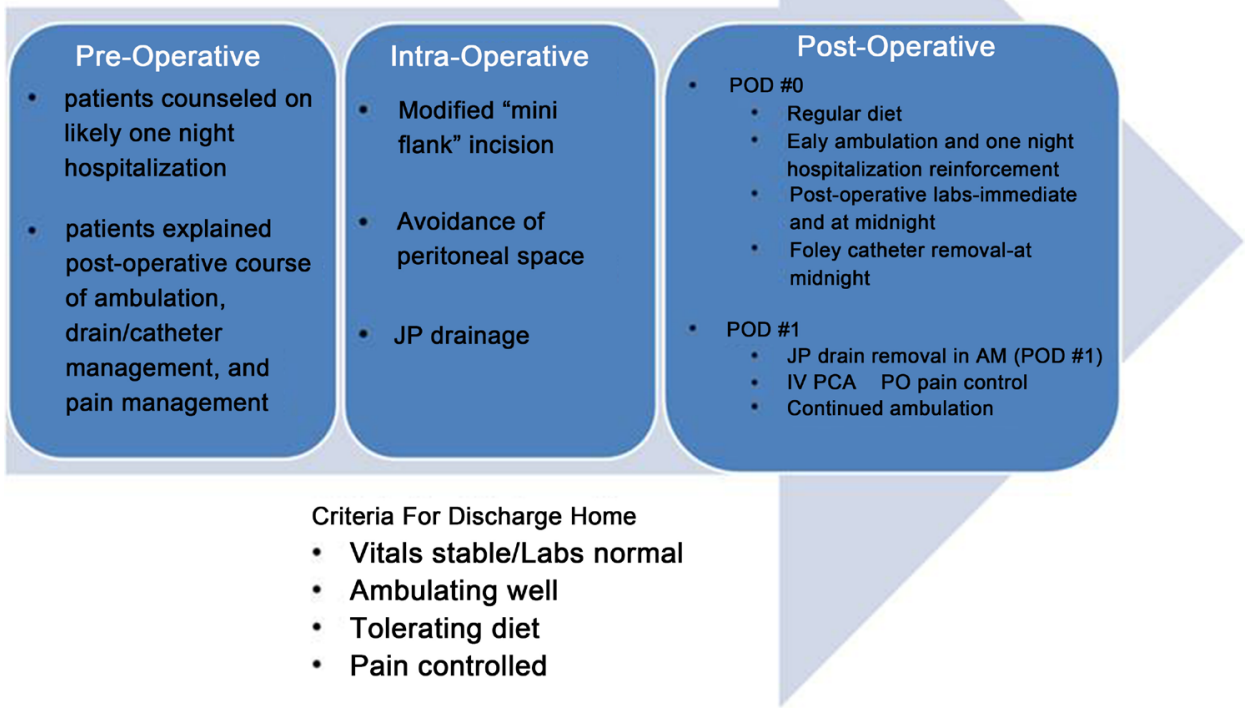

Figure 1. Rapid discharge pathway.

hospital one night and will recover if they follow a few basic steps. In the operating room, every attempt is made to limit incision size as this contributes the most to postoperative pain; in our experience, pain is the most common reason patients are not discharged after a one night stay. Therefore, we have adopted the use of and modified (through a slightly more posterior approach) the popularized mini-flank incision [7] [11] [18]. Additionally, every attempt is made to remain retroperitoneal, as ileus will also delay discharge if bowel is encountered. Every patient is left with a drain to be sure to detect any urine leaks in an effort to minimize missing complications.

Complication rates between OPN and RPN have not been shown to significantly differ. However, some individual studies have reported that RPN has lower rates of complications and shorter LOS, but despite this difference the overall cost is still higher than OPN [19]. Hospitalization costs significantly increase with complications, therefore it is important to recognize our rate of major post-operative complications, those believed to increase length of stay, was only $4 \%$, compared to $3 \%-24 \%$ (OPN) and $1 \%-9 \%(\mathrm{RPN})$ in other reported studies [1] [2] [20] [21]. More importantly, it again establishes that the procedure can be accomplished through a mini flank incision safely and patients may be discharged after a one night stay, even if not performed at a high volume center.

We recognize the inherent limitations of the study. It is a single institution retrospective design with a relatively small sample number in each arm that utilizes a descriptive analysis model to compare our open experience to other published robotic experiences. Each institution has widely variable costs in terms of equipment, staffing, and insurance 
reimbursement in different regions of the world. Our study only involves a single surgeon who performed over 75 OPN over a 3 year period. Although one can argue that a similar systematic approach is utilized for each case, it is obvious that with increased frequency, procedures may be performed faster and complications can certainly be avoided or handled expeditiously. Our reported LOS may actually be shorter than listed because we used check in and check out time as surrogates for true admission time and discharge times. Future studies are needed to examine the role of a newly instituted post-operative pathway to determine its efficacy in earlier discharge after OPN at lower volume surgical centers.

\section{Conclusion}

One night hospital stay for RPN is possible, but this is not the published norm [22]. While one night LOS for OPN is possible, it is not the predominant previously published outcome for patients. However, our purpose is not to prove that OPN is superior to RPN, it is only to demonstrate that a one night LOS can still safely be performed, thus leading to reduced costs. And when analyzing all available approaches, the open surgical approach is clearly still predominant in the community setting, so any method to further improve outcomes is necessary in an era where there are several modalities of treatment and cost is an important driver to the patient, doctor and hospital system.

\section{References}

[1] Lee, S., Oh, J., Hong, S.K., et al. (2011) Open versus Robot-Assisted Partial Nephrectomy: Effect on Clinical Outcome. Journal of Endourology, 25, 1181-1185. http://dx.doi.org/10.1089/end.2010.0670

[2] Masson-Lecomte, A., Yates, D.R., Hupertan, V., et al. (2013) A Prospective Comparison of the Pathologic and Surgical Outcomes Obtained after Elective Treatment of Renal Cell Carcinoma by Open or Robot-Assisted Partial Nephrectomy. Urologic Oncology, 31, 924-929. http://dx.doi.org/10.1016/j.urolonc.2011.08.004

[3] Mellon, M.J., Lucas, S.M., Kum, J.B.J., et al. (2013) A Comparison of Pathologic Outcomes of Matched Robotic and Open Partial Nephrectomies. International Urology and Nephrology, 45, 381-385. http://dx.doi.org/10.1007/s11255-013-0392-4

[4] Campbell, S.C., Novick, A.C., Belldegrun, A., et al. (2009) Guideline for Management of the Clinical T1 Renal Mass. The Journal of Urology, 182, 1271-1279. http://dx.doi.org/10.1016/j.juro.2009.07.004

[5] Patel, M.N., Bhandari, M., Menon, M., et al. (2009) Robotic-Assisted Partial Nephrectomy. BJU International, 103, 1296-1311. http://dx.doi.org/10.1111/j.1464-410X.2009.08584.x

[6] Dindo, D., Demartines, N. and Clavien, P.A. (2004) Classification of Surgical Complications: A New Proposal with Evaluation in a Cohort of 6336 Patients and Results of a Survey. Annals of Surgery, 240, 205-213. http://dx.doi.org/10.1097/01.sla.0000133083.54934.ae

[7] Diblasio, C.J., Snyder, M.E. and Russo, P. (2006) Mini-Flank Supra-11 ${ }^{\text {th }}$ Rib Incision for Open Partial or Radical Nephrectomy. BJU International, 97, 149-156.

http://dx.doi.org/10.1111/j.1464-410X.2006.05882.x

[8] Ficarra, V., Minervini, A., Antonelli, A., et al. (2014) A Multicenter Matched-Pair Analysis Comparing Robot-Assisted versus Open Partial Nephrectomy. BJU International, 113, 
936-941. http://dx.doi.org/10.1111/bju.12570

[9] Ghani, K.R., Sukumar, S., Sammon, J.D., et al. (2014) Practice Patterns and Outcomes of Open and Minimally Invasive Partial Nephrectomy since the Introduction of Robotic Partial Nephrectomy: Results from the Nationwide Inpatient Sample. The Journal of Urology, 191, 907-913. http://dx.doi.org/10.1016/j.juro.2013.10.099

[10] Patel, H.D., Mullins, J.K., Pierorazio, P.M., et al. (2013) Trends in Renal Surgery: Robotic Technology is Associated with Increased Use of Partial Nephrectomy. The Journal of Urology, 189, 1229-1235.

[11] Mano, R., Schulman, A., Hakimi, A.A., et al. (2015) Cost Comparison of Open and Robotic Partial Nephrectomy Using a Short Postoperative Pathway. Urology, 85, 596-604. http://dx.doi.org/10.1016/j.urology.2014.10.044

[12] Alemozaffar, M., Chang, S.L., Kacker, R., et al. (2013) Comparing Costs of Robotic, Laparoscopic and Open Partial Nephrectomy. Journal of Endourology, 27, 560-565.

http://dx.doi.org/10.1089/end.2012.0462

[13] Laydner, H., Isac, W., Autorino, R., et al. (2013) Single Institutional Cost Analysis of 325 Robotic, Laparoscopic, and Open Partial Nephrectomies. Urology, 81, 533-539. http://dx.doi.org/10.1016/j.urology.2012.07.104

[14] Lucas, S.M., Mellon, M.J., Erntsberger, L. and Sundaram, C.P. (2012) A Comparison of Robotic, Laparoscopic and Open Partial Nephrectomy. JSLS, 6, 581-587. http://dx.doi.org/10.4293/108680812X13462882737177

[15] Mir, S.A., Cadeddu, J.A., Sleeper, J.P. and Lotan, Y. (2011) Cost Comparison of Robotic, Laparoscopic and Open Partial Nephrectomy. Journal of Endourology, 25, 447-453. http://dx.doi.org/10.1089/end.2010.0510

[16] Benway, B.M. and Bhayani, S.B. (2011) Re: Cost Comparison of Robotic, Laparoscopic and Open Partial Nephrectomy. Journal of Endourology, 25, 1675-1676. http://dx.doi.org/10.1089/end.2011.0096

[17] Kim, S.P., Gross, C.P., Smaldone, M.C., et al. (2015) Perioperative Outcomes and Hospital Reimbursement by Type of Radical Prostatectomy: Results from a Privately Insured Patient Population. Prostate Cancer and Prostatic Diseases, 18, 13-17.

[18] Russo, P. and Mano, R. (2014) Open Mini-Flank Partial Nephrectomy: An Essential Contemporary Operation. Korean Journal of Urology, 55, 557-567. http://dx.doi.org/10.4111/kju.2014.55.9.557

[19] Yu, H., Hevelone, N.D. and Lipsitz, S.R. (2012) Use, Costs and Comparitive Effectiveness of Robotic Assisted, Laparoscopic and Open Urological Surgery. The Journal of Urology, 187, 1392-1399. http://dx.doi.org/10.1016/j.juro.2011.11.089

[20] Kim, S.P., Leibovich, B.C., Shah, N.D., et al. (2012) The Relationship of Postoperative Complications with In-Hospital Outcomes and Costs after Renal Surgery for Kidney Cancer. BJU International, 111, 580-588. http://dx.doi.org/10.1111/j.1464-410X.2012.11122.x

[21] Vittori, G. (2014) Open versus Robotic-Assisted Partial Nephrectomy: A Multicenter Comparison Study of Perioperative Results and Complications. World Journal of Urology, 32, 287-293. http://dx.doi.org/10.1007/s00345-013-1136-x

[22] Abaza, R. and Shah, K. (2013) A Single Overnight Stay Is Possible for Most Patients Undergoing Robotic Partial Nephrectomy. Urology, 81, 301-307.

http://dx.doi.org/10.1016/j.urology.2012.08.067 
Submit or recommend next manuscript to SCIRP and we will provide best service for you:

Accepting pre-submission inquiries through Email, Facebook, LinkedIn, Twitter, etc. A wide selection of journals (inclusive of 9 subjects, more than 200 journals)

Providing 24-hour high-quality service

User-friendly online submission system

Fair and swift peer-review system

Efficient typesetting and proofreading procedure

Display of the result of downloads and visits, as well as the number of cited articles

Maximum dissemination of your research work

Submit your manuscript at: http://papersubmission.scirp.org/ 